Gunther Teubner

Peer Zumbansen

\title{
Rechtsentfremdungen: Zum gesellschaftlichen Mehrwert des zwölften Kamels
}

(Zeitschrift für Rechtssoziologie 21, 2000, 189-215 und in Gunther Teubner (Hg.) Die Rückgabe des zwölften Kamels: Niklas Luhmann in der Diskussion über Gerechtigkeit. Lucius \& Lucius, Stuttgart 2000, 189-215)

I.

Oase in der Wüste oder Fata Morgana? Dort wo Jacques Derrida im gleißenden Licht der Wüstensonne die mythische Gewalt der Selbst(be-)gründung des Rechts erblickt, wo Hans Kelsen die Grundnorm des Rechts und Herbert Hart die ultimate rule of recognition beobachten, sieht Niklas Luhmann das Kamel des Khadis auf einer grünen Weide grasen. Allen geht es um die Frage der Letztbegründung des Rechts, für Luhmann jedoch heißt das, die inneren Paradoxien des Rechts, die problematische Beziehung des Rechts zu sich selbst, aufzudecken. Das zwölfte Kamel des Khadis steht nicht als Symbol für etwas anderes, sondern verkörpert selbst die entparadoxierenden symbolischen Operationen des autopoietischen Rechts. Es ist die lokalisierte Selbstreferenz des Rechts, die letztlich für die das Rechtssystem antreibende Dynamik von paralysierenden Paradoxien und befreienden Ausweichbewegungen verantwortlich ist.

Solche Überlegungen zur Autopoiese des Rechts und ihren Folgen sollen hier fortgesetzt werden, jedoch aus einer anderen Perspektive. Es geht jetzt nicht um die innere Selbstreferenz des Rechts und ihre Konsequenzen, sondern wie die äußeren Beziehungen des Rechts zu seiner gesellschaftlichen Umwelt zu fassen sind, wenn es als operativ geschlossenes System verstanden wird. Was ist der gesellschaftliche Mehrwert des zwölften Kamels? Und wie erfüllt die Gesellschaft ihre dem Recht geschuldete Pflicht - die Rückgabe des zwölften Kamels? Im Anschluß daran stellen sich schwierige Fragen der Adäquanz des Rechts zu den von inm geregelten sozialen Konflikten. Wie realistisch ist das Bild des richterlichen Kamels? Dann ist zu fragen, wie man in der Theorie der Rechtsautopoieis die problematische Beziehung zwischen der richterlichen Entscheidung und ihren Grundlagen faßt. Ist das zwölfte Kamel nur eine rhetorische Kunstfigur der Rechtsargumentation, welche die eigentliche Basis der Rechtsentscheidungen verbirgt? Oder "trägt“ das Kamel die richterliche Entscheidung in dem Sinne, daß sie wenn nicht determiniert so doch gerechtfertigt wird? Schließlich stellen sich Fragen der Gerechtigkeit eines selbstreferentiell geschlossenen Rechts. Verbürgt das zwölfte Kamel eine gerechte Lösung des Individualkonflikts? Und wird es den gesellschaftlichen Strukturen seiner Zeit gerecht? ${ }^{1}$

\footnotetext{
${ }^{1}$ Diese Frage nach der Einzelfallgerechtigkeit und der Gesellschaftsadäquität läßt sich auch für die konkrete juristische Lösung stellen, die das heutige Recht für de Erbteilungskonflikt zwischen den Brüdern bereitstellt. Interessanterweise stellt das moderne Erbrecht in einem Fall, in dem die Teilungsanordnung des Erblassers nicht „aufgeht“, den Parteien nicht nur ein Kamel, sondern gleich zwei Kamele zur Verfügung. § 2089 läßt die von der Teilungsanordnung nicht erfaßten Teile den Erben im Verhältnis ihrer Erbteile anwachsen. Darin steckt eine abstraktere Fassung der Lösung des Khadis, welche die Fiktion des zusätzlichen Kamels in eine generelle Rechtsnorm verwandelt. § 2088 aber ordnet an, daß der nicht erfaßte Teil ganz anders, nämlich nach den Regeln der gesetzlichen Erbfolge zu verteilen ist. Welche der beiden Lösungen gelten soll, entscheidet sich nach Auslegung des Erblasserwillens, ob die Erben die alleinigen Erben sein sollen oder nicht. Über die Zulässigkeit der zweiten Lösung, die höheren Variantenreichtum zuläßt, aber auch die Einheit des
} 
II.

Rechtsentfremdung ist der Tenor einer rechtssoziologischen Dauerkritik, die von den unterschiedlichsten Denkschulen - von Eugen Ehrlich und den Rechtsrealisten über normative Denker wie Hayek und Habermas und critical legal students bis zu jüngeren Dekonstruktionsunternehmern - am Recht geübt wird. Die Kritik gilt der Entfremdung des Rechts von seinen gesellschaftlichen und menschlichen Ursprüngen, seiner gewaltsamen Abstraktion von der Beziehung zwischen Ego und Alter. In ihren verschiedenen Varianten fordert die Rechtskritik Rückkehr zu dem, was sie jeweils als die Ursprünge des Recht ansieht: gesellschaftliche Normen, spontane Regelbildung, diskursive Rationalität oder dekonstruktive Gerechtigkeit. Besonders aufschlußreich erscheint Christies Formel: Recht als Enteignung von Konflikten. ${ }^{2}$ Das Recht selbst ist danach prinzipiell nicht in der Lage, soziale Konflikte ausreichend zu verstehen und in angemessener Weise zu lösen. In erster Linie liegt dies daran, daß das Recht über rechtliche Begrifflichkeiten und Verfahren die gesellschaftlichen Konflikte derart formalisiert, daß es sie letztlich ihrem Kontext des sozialen und moralischen Verständnisses der streitenden Parteien „enteignet“. Daher lautet die neue Formel: „Expropriiert die Expropriateure! Gebt die Konflikte den Menschen zurück!" Mit diesem suggestiven Slogan bringt Christie ein weitverbreitetes Unbehagen an rechtlicher Konfliktlösung, die als eigentlich inhuman, irrational und unsensibel gegenüber den Bedürfnissen der Beteiligten erfahren wird, zum Ausdruck.

Das zwölfte Kamel dagegen feiert die Enteignung des Rechts. Die Ursünde des Rechts wird zu seiner Kardinaltugend. In der Tat, wenn das Recht einen sozialen Konflikt unter höchst artifiziellen Verfahrensbedingungen und mit einer höchst artifiziellen Sprache rekonstruiert, wird der Ursprungskonflikt entfremdet, enteignet, disloziert, disseminiert, ja verzerrt und verfälscht. So gesehen hieße das zwölfte Kamel zu beobachten, sich überhaupt erst auf das Proprium des Rechts einzulassen. Das Recht selbst kann im Prinzip nicht genügend Empathie entwickeln, um die sozialen Konflikte „dort draußen“ zu verstehen. Stattdessen kann es sie nur in technische Rechtsfragen transformieren. Es rekonstruiert sie aber so, daß sie auf jeden Fall rechtlich entscheidbar werden. Und das heißt zugleich, daß die quaestio iuris wenig oder gar nichts mehr mit dem ursprünglichen sozialen Konflikt zu tun hat. ${ }^{3}$ Das Kamel des Khadi ist eben anders als die elf Kamele des Scheichs. Rechtsfälle können und sollen nicht das getreue Abbild sozialer Konflikte sein. Wie besonders die Debatte über Alternativen zum Recht deutlich gezeigt hat, kann das Recht weder ein adäquates Verständnis ihrer tieferen Ursachen, ihres lebensweltlichen Sinnes, ihrer Lösungsmöglichkeiten, noch ihrer sozialen Konsequenzen entwickeln. Das Recht ist keineswegs besonders gut geeignet, zwischenmenschliche Konflikte in einer die Beteiligten zufriedenstellenden Weise zu lösen. ${ }^{4}$ Verhandlung, Mediation, Schlichtung, Schiedsverfahren und Vergleiche werden der Natur von Konflikten, ihren Ursachen und den Bedürfnissen der Beteiligten sehr viel besser gerecht. Man kann sehr gut mit Konflikten umgehen, ohne sich überhaupt des Rechts zu bedienen. Kurz, wenn das Recht sozia-

Nachlasses in Frage stellt, herrschte unter Juristen jahrhundertelang ein erbitterterter Streit, der um die Bedeutung der rätselhaften römischen Rechtsregel nemo pro parte testatus pro parte intestatus decedere potest geführt wurde. Zur rechtshistorischen und Entwicklung und zum Zusammenhang mit Familien-, Wirtschafts- und Sozialstrukturen vgl. Becker, 2000 m.w.N.

2 Christie 1977.

$3 \quad$ Galtung 1965.

$4 \quad$ Fitzpatrick 1992. 
le Konflikte entscheidet, dann entfremdet es sie aufgrund seiner selbstproduzierten Fiktionen.

Dieser Entfremdungseffekt ist historisch variabel und nicht etwa ein universelles Attribut des Rechts selbst. Viele Rechtsordnungen sind in hohem Maße in gesellschaftliche Normen und Verhaltensweisen eingebettet und reflektieren unmittelbar die jeweils herrschenden religiösen oder politischen Orientierungen. Die Rechtsentfremdung, von der hier die Rede ist, ist dagegen das Ergebnis einer spezifischen historischen Konfiguration. Andererseits ist sie nicht Ergebnis absichtsvoller Planung oder rationalen Entwurfs. Sie beruht nicht auf politischer Entscheidung für den Aufbau einer Rechtsmaschinerie, die Konflikte auf die beschriebene Art zu entfremden hat. Vielmehr ist der Schlüssel zum Verständnis der Rechtsentfremdung das Phänomen des re-entry. ${ }^{5}$ Die These heißt, daß Konfliktentfremdung das typische Nebenprodukt einer doppelten Schließung des Rechts ist, die ihrerseits durch den re-entry von Rechtsunterscheidungen in diese selbst zustandekommt.

Gerichtsurteile, Legislativakte, aber auch Verträge, innerorganisatorische Normierungen und Standardisierungen zeichnen sich als Rechtsakte dadurch aus, daß sie - im Unterschied zu bloßen ökonomischen Transaktionen und politischen Kollektiventscheidungen das Symbol der Rechtsgeltung von einer Norm zur anderen übertragen. Die Geltungsübertragung findet ausschließlich in rekursiven Verkettungen von Gerichtsurteilen, Legislativakten und privatautonomen Entscheidungen auf der Grundlage binärer Codierung (Recht/Unrecht) statt. Diese Verkettung von Rechtsakten stellt die erste Schließung, die operative Schließung, des Rechts dar. ${ }^{6}$ Die problematischen Aspekte operativer Schließung sind, wenn sie nicht anderweitig kompensiert werden, leere Tautologien, unproduktive Selbstreferenzen, rigide Formalismen und die Isolierung des Rechts von seiner sozialen Umwelt. Kritiker der operativen Schließung lenken entsprechend die Aufmerksamkeit auf die Präsenz sozialer Phänomene im Innern des Rechts und versuchen auf diese Weise, die Grenzen des Rechts aufzubrechen. Die Zauberformel heißt communal justice. ${ }^{7}$

Tatsächlich aber ist die Rechtsevolution in anderen Bahnen verlaufen. Die operative Geschlossenheit des Rechts wurde nicht durch verstärkte soziale Einbettung der Rechtsnormen in Sozialstrukturen kompensiert. Das genaue Gegenteil geschah: eine Verdoppelung der Schließung. ${ }^{8}$ Die Schließung der Rechtsoperationen wurde durch die Schließung rechtlicher (Selbst-)Beobachtungen komplettiert. Sie vollzog sich in dem Moment, in dem Argumentatioen ad hoc und ad hominem im Rechtsverfahren ausgeschlossen wurden und an ihre Stelle die Berufung auf spezielle Rechtsmaterialien - Präzedenzien, gelehrte Meinungen, Rechtsnormen und Rechtsprinzipien - traten. Erst dann konnte es dazu kommen, dass sich das Recht mehr oder weniger erfolgreich von direkten sozialen Pressionen und von diffusen Abhängigkeiten, die auf Klientelismus, Verwandtschaft, sozialem Status oder Freundschaft beruhen, isoliert. ", The artificial reason of law“, den Sir Edward Coke gegen die politischen Interventionen des Königs ins Feld führt, machen es gegenüber den unmittelbaren Geltungsansprüchen moralischer Argumente, wirtschaftlicher Erwägungen, politischer Zwecke und common-sense-Weisheiten autonom.

Zum Begriff des re-entry allgemein Spencer 1972, 56 f.; 69 ff.; Luhmann 1993a; 1997, 179 ff.; Esposito 1993.

Cotterrell 1995, Kap. 5, 15.; zur Kritik operativer Schließung vgl. auch Kerchove/Ost 1992.

Zur doppelten Geschlossenheit als einer notwendigen Voraussetzung von Selbstorganisation, s. insb. Foerster 1993; Luhmann 2000, Kap. 7.; Baecker 1993. 
Wie aber sollte ausgerechnet „the artificial reason of law“ die Probleme operativer SchlieBung kompensieren? Der Grund hierfür ist im re-entry, der auf eigentümliche Weise AuBerrechtliches ins Recht integriert, zu suchen. Während rechtliche Operationen bereits aufgrund ihrer Sequentialisierung die Grenze zwischen Recht und Nicht-Recht, d.h. die Grenze zwischen spezifischen Rechtsakten und anderen Formen sozialer Kommunikation, etablieren, nehmen rechtliche Selbstbeobachtungen genau diese Unterscheidung zwischen Recht und Nicht-Recht auf, nun aber innerhalb des symbolischen Raum des Rechts. Um Mißverständnissen vorzubeugen, hier geht es nicht um die Differenz Recht/Unrecht, die binäre Codierung des Rechts, die wir oben benannten, mit deren Hilfe die erste Schließung des Rechts überhaupt erst bewerkstelligt wird. Die Codierung Recht/Unrecht konstituiert in ihrem Gebrauch die Grenze zwischen Recht/Nicht-Recht und ist damit für die operative Schließung verantwortlich ist. Demgegenüber führt der re-entry der Unterscheidung Recht/Nicht-Recht innerhalb des Rechts zur observationellen SchlieBung. ${ }^{10}$ Der re-entry eröffnet rechtlichen Argumenten zwei Optionen: Entweder beziehen sie sich auf interne Rechtsoperationen, oder sie beziehen sich auf externe soziale Ereignisse. Und hier, in diesem zweiten Aspekt, vollzieht sich nun die Kompensation der operativen Schließung. Sobald die Unterscheidung von Recht/Nichtrecht (im Sinne von rechtlich/außerrechtlich) in die Sequenz rechtlicher Operationen wiedereingeführt wird, kann die autonomisierte rechtliche Argumentation selbst auf „Außerrechtliches“ dadurch zurückgreifen, daß sie zwischen Geltung und Faktizität, zwischen internen Rechtsakten und externen Sozialereignissen, zwischen Rechtsbegriffen und sozialen Interessen, zwischen internen Realitätskonstruktionen des Rechtsprozesses und denen sozialer Prozesse unterscheidet. Wobei zu betonen ist, daß dies alles innerrechtliche Unterscheidungen sind. Dies ist als die paradoxe Errungenschaft der doppelten Schließung des Rechtssystems anzusehen. Während regelproduzierende Rechtsakte und regelverbindende Rechtsargumente je in ihrem geschlossenen Verkettungskreislauf eingebunden bleiben, führt die intern benutzte Unterscheidung zwischen Selbst- und Fremdreferenz dazu, dass das Recht, das sich zunächst kraft operativer Schließung von der Umwelt unabhängig gemacht hatte, sich nun wieder auf eigentümliche Weise von seiner Umwelt abhängig macht. Um dies noch einmal zu unterstreichen, diese Umwelt ist eine „hergestellte“, nicht eine „natürliche“, „wirkliche“ Umwelt. ${ }^{11}$ Und die höheren Freiheitsgrade, welche durch diese interne Rekonstruktion von Realität im Vergleich zu einer Eins-zu-eins-Korrelation von Internem und Externem gewonnen werden, steigern die Chancen für eine gelingende Kompensation der ersten Schließung.

Ein re-entry hat jedoch seinerseits drastische Folgen. ${ }^{12}$ Sobald die Unterscheidung zwischen Recht und Nicht-Recht in das Recht selbst wiedereingeführt wird, entsteht eine erhebliche epistemische Konfusion (à la Magritte: „Dies ist keine Pfeife“) über den Realitätsstatus der fremdreferentiellen Beobachtungen des Rechts (die „Rechtstatsachen“). Das zwölfte Kamel ist ein Kamel ist ein Kamel, nicht anders als die übrigen elf Kamele? Natürlich handelt es sich um eine reine Fiktion, aber der Khadi muss die Illusion aufrecht erhalten, dass sein Kamel real ist. Ergebnis des re-entry ist die Schaffung eines imaginären Raums innerhalb des Rechts, der sich in seiner Selbstbeobachtung für real hält und für real halten muß. Das Recht kann nicht anders als Fiktionen über die Außenwelt zu schaffen, die es aber gleichzeitig als harte Realitäten behandeln muss. Die Oase, in der das zwölfte Kamel weidet, ist nur eine Fata morgana des Rechts, und dennoch kann der Richter die streitenden Brüder zur Kamelausleihe in diese Oase schicken und mit dieser Auf-

$10 \quad$ Luhmann 1993b, 67ff., 338ff.

$11 \quad$ Weick 1979; Varela 1992, $235 \mathrm{ff}$.

12 Hierzu besonders Spencer Brown 1972, 56f.; $69 \mathrm{ff}$. 
forderung ganz erstaunliche reale Wirkungen zeitigen. Und hier liegt der eigentliche Grund für die Unausweichlichkeit der Entfremdung sozialer Konflikte. Mit anderen Worten, hier zeigt sich, dass die vielkritisierte Entfremdung des Rechts gerade nicht etwas ist, das man, wenn man sich nur ordentlich Mühe gibt, überwinden könnte, sondern das zum Selbstverständnis des Rechts gehört.

Die Enteignung sozialer Konflikte ist somit nicht einfach ein banaler Diebstahl. Diesen Akt mißversteht man gründlich, wollte man darin lediglich eine Usurpation sozialer Kompetenzen durch die Rechtsexperten erkennen, die ihre Spezialsprache als Machtinstrument einsetzen. ${ }^{13}$ Im vorliegendem Erbstreit führt die Rechtsentfremdung dazu, daß sich die Anzahl der Scheichkamele vergrößert, was eine produktive Aufteilung des Erbes möglich macht. Allgemeiner ausgedrückt steigert die Entfremdung durch das Recht die Möglichkeiten sozialer Sinnproduktion. Gerade durch ihre sinnverfälschenden Transformationen eröffnet sie neue, unbekannte, ohne sie nicht zugängliche Sinnwelten, wodurch sie traditionelle Denkbahnen mit ihren auf bisherigen Erfahrungen basierenden Erwartungen erweitert. Hier zeigt sich eine deutliche Parallele zwischen Recht und Kunst, da beide eine zweite Realität erzeugen, in unserem Fall die Fata morgana des Khadis, in der jedenfalls mehr und andere Kamele leben als in der wirklichen Welt der Wüste. In diesem Lichte betrachtet haben kritische Rechtssoziologen eine verblüffende Ähnlichkeit mit einem Herrn Beckmesser, der an dem famosen Künstler Hans Sachs auszusetzen hatte, dieser lasse den Sinn für das Wirkliche vermissen. Innen entgeht die Besonderheit des Rechts und der Kunst, die darin besteht, dass hier Konstrukte gebaut werden, die zuvor gar nicht vorstellbar waren. Gäbe es nicht die Kunstsprache der Rechtsdogmatik, so wäre es nicht möglich, den Konflikt im Rechtssinne zu rekonstruieren, die andersartige quaestio iuris zu stellen, die ihrerseits in eine Sequenz von Tatfragen und Rechtsfragen aufgelöst wird, und neue, bisher nicht dagewesener Argumentationen, Kriterien und Regeln zu finden. Nach dem Verbot der Rechtsverweigerung ist die Produktivitätssteigerung durch Rechtsentfremdung besonders deutlich geworden, da eine Rechtsentscheidung selbst dann noch garantiert ist, wenn die Beweiserhebung in einem non liquet enden oder die Rechtsargumentation zu keinem eindeutigen Ergebnis führen sollte.

Und unter bestimmten Umständen kann es sogar passieren, dass das zwölfte Kamel aus der Welt des Khadis in die wirkliche Welt der Beduinen gelangt und dort die Zahl ihrer Handlungsoptionen vergrößert, was wiederum die Chancen einer besseren Konfliktlösung steigern mag. Sicher gibt es hier keinen Automatismus, denn nur in seltenen Momenten wird aus Rechtsentfremdung mehr als Rechtsenteignung. Als eine gelungene Rückübersetzung - von einem gesellschaftlichen Konflikt zu einer Rechtsfrage und von dort zurück zu einem sozialen Ereignis - vermag dieser Vorgang, gleichsam als ein Zusammentreffen glücklicher Umstände, neue Handlungsmöglichkeiten in dieser Welt zu eröffnen.

Wenn aber das Kamel das rechtliche proprium verkörpert, was hat es dann mit diesem proprium auf sich? Die Hilfe des Khadi für die Söhne des Scheichs besteht ja nicht darin, daß er das inm gestellte Problem auf einer höheren Rechtsebene interpretiert. Ebensowenig entscheidet er aufgrund von Prinzipien der iustitia distributiva, deren Regeln zu einer Verfeinerung sozialer Handlungsformen führen. Stattdessen leiht der Khadi den Scheichssöhnen eine Fiktion - und genau dies ist das rechtliche proprium. Und die Nichtübereinstimmung mit der Normalrealität ist das Entscheidende. Die Stunde des Rechts ist in dem Moment gekommen, in dem Konflikte sich mit Hilfe normale Realitätskonstruktio- 
nen als nicht lösbar herausstellen. Im Recht kann man dann eine zusätzliche, zugegebenermaßen unwirkliche, künstliche, erfundene Welt betreten, die diese Blockierung auflöst. Fiktionalisierung eröffnet die Möglichkeit, dass Konflikte im Zustand ihrer moralischen und sozialen Unlösbarkeit entscheidbar werden, wenn auch nur in der imaginären Welt des Rechts.

Offensichtlich sind damit die üblichen Vorstellungen über die Sozialadäquanz des Rechts auf den Kopf gestellt. Gleichzeitig schließen sich an diesem Punkt neue Forschungsfragen und andersartige politische Herausforderungen an. Wenn es nicht mehr darum geht, das Recht für das Selbstverständnis der involvierten Parteien zu öffnen oder es für geltende soziale Normen, moralische Werte und Gemeinschaftsvorstellungen empfänglich zu machen, dann heißt die Formel: gegen common sense ist der Konflikt zu rekonstruieren. Nicht die Rückbindung rechtlicher Entscheidungen an Sozialnormen macht den Mehrwert der rechtlichen Rekonstruktion aus, sondern ihre Entfremdung von ihnen. Dann aber lautet die entscheidende Frage auch nicht länger, ob diese Fiktionen den inneren Komplexitäten des jeweiligen Konflikts korrespondieren. Statt dessen geht es um die Arbeitsweise der Fiktionen selbst, also darum, wie sie die Rechtsarbeit beeinflussen, in welche Richtung sie sich entwickeln und zu welchen gesellschaftlichen Umwelten sie selektive Affinitäten herausbilden.

\section{III.}

Determiniert dann die Fiktion des zwölften Kamels die Entscheidung? Vermag sie die Teilung des väterlichen Erbes zu rechtfertigen? Es scheint, als müßte man an dieser Stelle in der Dauerdebatte zwischen Bestimmtheit und Unbestimmtheit rechtlicher Argumentation Partei ergreifen, doch führt re-entry auf eine dritte Position. Sobald rechtliche Operationen ihrerseits zwischen Recht und Nichtrecht unterscheiden, entsteht wie gesagt eine Situation unauflösbarer Unbestimmtheit innerhalb des Rechts selbst. Das Recht kann mit den inm zur Verfügung stehenden Argumenten die Unbestimmtheit nicht auflösen. Dies zwingt das Recht zur internen Differenzierung zweier unterschiedlicher Typen rechtlicher Operationen: Rechtsentscheidungen, die den Geltungstransfer symbolisieren, und Rechtsargumente, die die Beziehung zwischen Redundanz und Varietät im Recht regulieren. ${ }^{14}$ Mit dieser Differenzierung ist gesichert, daß die durch Selbstbeobachtung erzeugte Unbestimmtheit stets durch Entscheidungen aufgelöst werden kann. Es entstehen zwei unterschiedliche Kreisläufe rechtlicher Kommunikationen: Entscheidungsverkettungen und Argumentationsverkettungen. Ergebnis dieser Differenzierung ist, daß zwar beide Kreisläufe eng aufeinander bezogen sind, daß aber keiner den anderen determinieren kann. So werden Rechtsentscheidungen aufgrund ihrer Bindungskraft regelmäßig in neue Rechtsargumente transformiert, doch können sie nicht endgültig den Fluß rechtlichen Argumentierens determinieren. Rechtsargumente wiederum sind zweifellos unverzichtbare Elemente der Rechtsentscheidung, sie sind aber prinzipiell nicht in der Lage, Rechtsentscheidungen zu determinieren oder zu rechtfertigen.

Dies richtet sich direkt gegen Theorien, die auf Rechtfertigung durch rationale Argumentation im Recht bauen und sich zur Herstellung von Rechtsentscheidungen auf die intrinsische Kraft vernünftiger Motive verlassen. Ihr blinder Fleck liegt in der unauflösbaren Unbestimmtheit des Rechts. ${ }^{15}$ Gleichzeitig richtet sich dieser Ansatz aber gegen dezisionisti-

$14 \quad$ Luhmann 1995.

15 Siehe die Diskussion zur Unbestimmtheit des Rechts bei Habermas 1992, Kapitel 5; vgl. dazu auch Günther 1988, Kap 4; 3. 
sche und rechtsrealistische Ansätze und gegen critical legal studies. ${ }^{16}$ Deren blinder Fleck liegt in der Rolle von Rechtsargumenten. Während sie zurecht die unausweichliche Unbestimmtheit des Recht, die durch kein rechtliches Argument endgültig reduziert werden kann, herausstellen, sind sie nicht in der Lage, ein angemessenes Verständnis von juristischer Argumentation zu entwickeln. Wie erklärt sich die Überlebensfähgkeit juristischer Argumentationspraxis nach der rechtsrealistischen Entzauberung, nach der dezisionistischen Demystifikation, nach dem trashing der critical legal studies? Es erscheint wenig plausibel, rechtliches Argumentieren zur bloßen Maskerade zu erklären, die der Verdeckung von Machtstrukturen dient oder der Antizipierung von Konsens unter den Entscheidungseliten.

Der re-entry des zwölften Kamels verweist darauf, dass juristische Argumentation den Konflikt zwar nicht entscheidet, aber dennoch Entscheidendes bewirkt. Juristisches Argumentieren transformiert Differenzen. Es verwandelt die bestehende Entscheidungsalternative in eine neue. Für die oben angesprochene Entfremdung des sozialen Konflikts ist also gerade die Rechtsargumentation verantwortlich. Juristisches Argumentieren entscheidet nicht. Es rechtfertigt nicht. Es hat auch nichts zu verbergen. Es transformiert nur Entscheidungsalternativen, dieses aber drastisch. Eine Entscheidung bleibt nach wie vor notwendig, aber nach der Rechtsargumentation ist die sich präsentierende Entscheidungsalternative eine andere geworden.

Welchen Unterschied macht es, wenn ein Sozialkonflikt durch juristische Argumentation in einen zu entscheidenden Rechtskonflikt, also in einen Konflikt zwischen unterschiedlichen semantischen Artefakten des Rechts, überführt wird? Geht es dabei bloß um die bekannte Problematik der Subsumtion eines Lebenssachverhaltes unter die allgemeine Norm des Gesetzes? Suchen wir vielleicht nach einer neuen „goldenen Regel“, nach einem kategorischen Imperativ, einem weiteren Schleier des Nichtwissens? Aus der Sicht der Rechtsautopoiesis handelt es sich um etwas anderes; die Rechtsentfremdung, von der hier die Rede ist, bewirkt eine andere Transformation als die einer ethischen Generalisierung. Ein Teil der Antwort heißt: Selbstreferenz des Rechts. Die rekursive Anwendung rechtlicher Operationen auf die Ergebnisse von rechtlichen Operationen läßt ein artifizielles Netz von Begriffen, Regeln und Prinzipien entstehen, das sich, je elaborierter es mit der Zeit wird, desto weiter von den Partikularitäten einer konkreten Fallsituation entfernt. Diese endlose Praxis von Gleich-oder-Ungleich-Behandlungen stellt im Kern den Entfremdungsmechanismus dar. Was gleich ist, gleich zu behandeln, und was ungleich ist, ungleich, setzt eine sich ständig fortsetzende Reihe von Unterscheidungen in Gang. Es handelt sich um einen generativen Mechanismus, in den Worten von Foersters um eine „historische Maschine“17, die eine endlos anwachsende Komplexität in der Welt rechtlicher Fiktionen herbeiführt.

Doch ist dies nur die halbe Wahrheit. Sie bleibt bei der Beschreibung interner Selbstreferenz und rekursiver Anwendung früherer Entscheidungen auf neue Fallkonstellationen stehen. Die andere Hälfte der Entfremdungseffekte rührt vom Dauerbeschuß gesellschaftlicher Irritationen her, dem das Rechtssystem bei der Konfliktlösung ausgesetzt ist. Koevolutionäre Kontakte mit externen Sozialprozessen dirigieren die Transformationen von Rechtssemantiken, Regeln, Konzepten, Prinzipien und Lehrsätzen in eine Richtung, die nicht mehr nur durch die interne Rekursionsgeschichte von individuellen Fallerfahrungen bestimmt ist. Die für die Rechtsentfremdung typische Inkongruenz von Rechtsdogmatik 
und Individualkonflikt, die Nichtübereinstimmung von Rechtsnormen und Fallkonstellation, ist weitgehend bestimmt durch die Kovariation der Rechtsdogmatik mit individualkonfliktfernen, sich permanent wandelnden Sozialstrukturen. ${ }^{18}$

Freilich weisen die inneren Strukturen dieser ko-evolutionären Dynamik große historische Unterschiede auf. So ist es heute nicht mehr möglich, die Ko-evolution rechtlicher und sozialer Institutionen als Ergebnis von spontaner Regelbildung und Gewohnheitsrecht zu beschreiben. Die entscheidende Rolle spielen institutionalisierte Produktionsregimes. ${ }^{19}$ Dieser Begriff bezeichnet Verknüpfungen zwischen autonomen sozialen Systemen, zwischen Recht, Wirtschaft, Politik, Erziehung und Wissenschaft. Produktionsregimes evolvieren nicht selbst zu autopoietischen Systemen mit eigenen Elementen, Strukturen und Grenzen. In der Form struktureller Kopplungen ${ }^{20}$ sind Produktionsregimes lediglich Konfigurationen sehr heterogener Komponenten, Hybride zwischen Recht und Gesellschaft. Als solche sind Produktionsregime weder Funktionssysteme noch formale Organisationen. Ebensowenig sind sie Interaktionen im technischen Sinne der Systemtheorie ${ }^{21}$, sondern Institutionen der Kopplung anderer Sozialsysteme. ${ }^{22}$

Die Eigenprozesse der Produktionsregimes sind dafür verantwortlich, auf welche Weise Rechtsnormen mit verschiedenen Sozialstrukturen ko-variieren. ${ }^{23}$ Im Unterschied zu einer einheitlichen Sozialevolution nach dem Muster der natural selection, die durch diffusen Umweltdruck auf soziale Institutionen durch verschiedene Selektoren gekennzeichnet wäre, sind an der Ko-evolution innerhalb eines Produktionsregimes mehrere operational geschlossene Systeme beteiligt, welche ihre je eigenen spezifischen Evolutionsmechanismen aufweisen. Jedes von ihnen folgt einem anderen Muster von Variation, Selektion und Retention. Innerhalb eines Produktionsregimes verlaufen parallel zueinander mehrere autonome evolutionärer Prozesse, welche sich wechselseitig im Wege der Ko-evolution beeinflussen, ohne daß sie dabei ihre Autonomie verlieren. Es gibt entsprechend keine einheitliche Evolution des Produktionsregimes selbst, sondern die gleichzeitige Entfaltung mehrerer unterschiedlicher evolutionärer Dynamiken. Jeweils unabhängige evolutionäre Mechanismen innerhalb der autopoietischen Systeme des Rechts, der Wirtschaft und der Politik, der Erziehung und der Wissenschaft beeinflussen sich wechselseitig und zwingen inre jeweiligen Institutionen in einem Produktionsregime auf einen idiosynkratischen Evolutionspfad. Gleichzeitig bildet das Produktionsregime spezifische Kanäle der Ko-evolution aus, die für das Muster der wechselseitigen Beeinflussung verantwortlich sind. Für Rechtstheorie und Rechtsdogmatik folgt daraus, daß ihre Entwicklung nicht auf einer der Geschichte des common-law vergleichbaren und durch die Verarbeitung bestimmter sozialer Konflikte gekennzeichneten Bahn verläuft. Rechtstheorie und Rechtsdogmatik werden bestimmt durch ko-evolutionäre Prozesse innerhalb des Produktionsregimes, die das Recht in eine enge Kompatibilitätszone mit wirtschaftlichen, politischen und anderen nichtrechtlichen Institutionen überführen.

Im Produktionsregime wird das Netzwerk juristischer Operationen externen Irritationen ausgesetzt, die regelmäßig aus ganz anderen Kontexten stammen als die der dem Richter

Zur Irritation des operativ geschlossenen Rechtssystems durch gesellschaftliche Prozesse Teubner 1989, Kap. 4, 5; Luhmann 1993b, Kap.12.

Zur systemtheoretischen Reformulierung des Begriffs Teubner 1999a.

Siehe hierzu insb. Maturana/Varela 1987, $103 \mathrm{ff}$.

Vgl. zu diesen Unterscheidungen Luhmann 1975 - Interaktion, Organisation, Gesellschaft.

Teubner 1995.

Zur Idee der Produktionsregimes vgl. besonders Soskice 1997; zum Verhältnis von Produktionsregimen und Recht siehe Teubner 1998a; 1999a. 
vorgelegten Individualkonflikte. Die Dynamik dieser externen Irritationen führt offenbar unausweichlich dazu, daß die Individualkonflikte mit den Rechtsnormen, die zu ihrer Lösung formuliert werden, nicht mehr kongruent sind. Denn bei den Evolutionsprozessen innerhalb eines Produktionsregimes geht es nicht blo $ß$ um die graduelle Anpassung von bereits bestehenden Rechtsnormen an die Sozialwirklichkeit. Sondern es handelt es sich um autonome Prozesse sozialer Normproduktion, die in neue Rechtsnormen umgesetzt werden. Bei dieser Transformation sozialer Normen in Rechtssätze kommt es zu einer Infiltration heterogener, partikularistischer Rationalitäten und Geltungsansprüche in das auf Individualkonfliktlösung ausgerichtete Recht, welches zur Ausübung einer wirksamen Eingangskontrolle kaum in der Lage ist. Die Maschinerien der außerrechtlichen Rechtsproduktion, die der inneren Logik ausdifferenzierter Sozialbereiche gehorchen, finden sich in formalen Organisationen, informellen Netzwerken und Standardisierungsverfahren, die ihrerseits in enger Konkurrenz zur gesetzgeberischen und vertragsrechtlichen Rechtssetzung stehen. ${ }^{24}$

Der Rechtsanwendungsprozess kann diese externen Normprodukte nicht ignorieren oder sie als fremd abstoßen. Er kann sie nur „durchprozessieren“, in dem er sie einem Gerichtstest unterwirft. In diesem Verrechtlichungsvorgang wird innen rechtliche Gültigkeit verliehen, indem sie zu früherer Rechtsprechung, zu Gesetz und Verfassung ins Verhältnis gesetzt werden. Gleichzeitig werden ihnen aber im Zuge dieser „Verträglichkeitsprüfung" die für die Konfliktlösung benötigten Kriterien entnommen.

Damit zeitigt die Konfliktenteignung, von der wir oben gesprochen haben, einen doppelten Effekt: Zur konkreten Fallsituation wird Distanz aufgebaut und zugleich wird der Kontakt mit externen Produktionsregimes intensiviert. Der ko-evolutionäre Prozeß, der die interne Rekursion von Rechtsakten den Irritationen externer Produktionsregime aussetzt, produziert somit normative Strukturen, durch die - obwohl oder eher: gerade weil sie sich zum Ursprungskonflikt orthogonal verhalten - der einzelne Konflikt quasi aus der Perspektive einer „neutralen Instanz“, eines „unbeteiligten Dritten“ oder „unbefangenen Beobachters“ eingeschätzt werden kann. Mit Blick auf unseren Fall heißt dies: Das zwölfte Kamel kam nicht zur Schlichtung des Streits zwischen den Brüdern ins Spiel. Dies kann es auch gar nicht, weil es einem gänzlich anderen Kontext entstammt, in dem arithmetische Kalkulationen die Teilung an sich unteilbarer Zahlen erlauben.

Man gewinnt den Eindruck, als sei heute die Kluft zwischen dem, was auf der einen Seite zur konkreten Konfliktlösung erforderlich erscheint und was auf der anderen Seite an Rechtsnormen in der Ko-evolution mit anderen Institutionen innerhalb eines Produktionsregimes entsteht, enorm. Was hat es mit der konkreten Bedürfnissen der sich um ihr Geld streitenden Parteien zu tun, daß der Ausgang ihres Prozesses von der jüngsten parteipolitischen Initiative zur Wettbewerbspolitik abhängt? Warum sollte einem Verkehrsunfallopfer damit geholfen sein, daß die Frage seiner Entschädigung im Deliktsrecht mit Hilfe ökonomischer Theorie zur Allokationseffizienz gelöst wird? Die Konfliktlösung ist nicht Ergebnis der Adäquanz, sondern der Diskrepanz zwischen Regeln politischer, ökonomischer oder technischer Rationalität einerseits und den Interessenlagen des Individualkonflikts andererseits. Zwar ist es gerade die Inkongruenz der Perspektiven Prozessführung versus Produktionsregime -, die die Lösung eines unentscheidbaren Falles möglich macht. Das heißt aber nur, daß die Parteien ihr Urteil bekommen. Die Urteilsgründe können sie vergessen. 
Und welchen Preis zahlen die streitenden Parteien, die ja nicht nur eine Rechtsentscheidung, sondern ihr Recht suchen? Was bedeutet es für sie, daß sie das Kamel zurückgeben müssen? Sie investieren ihre Streitenergien, die dann durch die Argumentationsmaschinerie des Rechts zu ganz anderen Zwecken benutzt werden. Der Streit der Parteien ist nur noch der Anlaß zur Produktion je neuer abstrakter Regeln und Prinzipien, welche freilich ohne die Teilnahme von Kläger und Beklagtem - in entfernten Produktionsregimes erzeugt werden. Mit ihrem Rechtsstreit opfern die Parteien Zeit, Energie und Geld, um einem Produktionsregime zu rechtsverbindlichen Normen zu verhelfen. Dies also bedeutet Allahs Befehl an die Brüder, dem Richter das Kamel zurückzugeben. Und der Khadi macht mit der Kameltransaktion ein gutes Geschäft. Angesichts dessen könnte der Titel unser Beitrag ebensogut „Rechtsausbeutung“ heißen.

\section{IV.}

Damit haben wir die Perspektive verändert. Rechtsausbeutung heißt, daß das Recht die Streitsucht der Parteien benutzt, um die zukünftige Normenproduktion zu steigern. In seiner Weisheit und Großzügigkeit weiß der Khadi genau, wie er sich den Streit der Brüder über das Vatererbe zu Nutze machen kann. Indem er ihnen aufgibt, das Kamel zu füttern, zu pflegen und später an ihn gut genährt zurückzugeben, verpflichtet er die Brüder, die innen zur Verfügung stehenden Streitressourcen für Zwecke der Normenproduktion zu mobilisieren. Genau dies ist das Ergebnis der Streitmaxime im Gerichtssaal. Das „Durchprozessieren" von Rechtsnormen macht es möglich, die Rechtsverbindlichkeit von unterschiedlichen Normprojektionen der Produktionsregimes autoritativ zu entscheiden. Durch die Erprobung von Argumentationsschemata im Parteienprozess werden die Normen eines Produktionsregimes der Feuertaufe des Gerichtstests ausgesetzt. Damit produzieren die Streitparteien nolens volens einen bedeutender Mehrwert für das Produktionsregime. $\mathrm{Daß}$ sie ihren Konflikt gerichtlich austragen, ist verantwortlich dafür, daß die ursprüngliche Bedeutung normativer Strukturen in Verträgen, Standards und Regelbildungen, wettbewerblichen Marktprozessen, intra-organisatorischen Regeln und politischen Gesetzen einschneidend transformiert werden. Die individuelle Prozeßführung vor Gericht ist in der Tat der Königsweg des Rechts, auf dem es zu verbindlichen Entscheidungen darüber gelangt, welche von den miteinander konkurrierenden Normen im Produktionsregime ausgewählt wird. Im Gerichtsprozeß gelingt es dem Recht, Konflikte zwischen vertraglichen Verpflichtungen, Standards und Gesetzesregeln aufzulösen, Rechtssätze von Sozialnormen, politischen Zielen und wirtschaftlichen Erwartungen, denen kein Rechtsstatus zukommt, zu trennen, Ambivalenzen durch eine bindende Interpretation aufzulösen. Gleichzeitig entstehen freilich neue Auseinandersetzungen und unterschiedliche Interpretationsmöglichkeiten, welche auf ihre Weise die Dynamiken rechtlicher und sozialer Institutionen innerhalb der Produktionsregime weiter antreiben. Im Gerichtsverfahren, aber auch schon durch seine Antizipation, können somit die Produktionsregimes von den Effekten der Verrechtlichung - beträchtlichen Zugewinnen an Erwartungsklarheit und -stabilität - profitieren.

Der Preis, der für diese Vorteile zu zahlen ist, ist erneut Rechtsentfremdung, die Nichtübereinstimmung der ursprünglichen Perspektiven des Produktionsregimes mit dem endgültigen Normenmanterial. Nur geht es diesmal zu Lasten des Produktionsregimes. Jetzt ist es die individuelle Prozeßführung vor Gericht, welche die guten Absichten der Produktionsregimes ruiniert. Nach den Prognosen der Evolutionsökonomik sollte ein wettbewerblich strukturierter Markt über eine Periode mehrfach wiederholter Transaktionen transaktionelle und organisatorische „Routinen“ hervorbringen, welche als effiziente Normen 
des Produktionsregimes gelten können. ${ }^{25}$ In dem Moment aber, in dem diese Routinen in einem Individualrechtsstreit vor Gericht durchprozessiert werden, werden sie von der ökonomischen Rationalität abgekoppelt. Im Gehedder des vor Gericht ausgetragenen

Rechtsstreites - durch Interessenabwägung, Policy-Argumente, Normkonsistenz- und Verfassungsmäßigkeitsprüfungen und nicht zuletzt durch die Berücksichtigung aller Umstände des Einzelfalles - wird die ursprüngliche Marktorientierung der Norm unweigerlich kontaminiert. Der Effizienzgewinn der marktseligierten Routinen geht im Prozess ihrer Kompatibilisierung mit einer Vielzahl von rechtlich relevanten Aspekten verloren. Und wo die sogenannte relitigation, also die hartnäckig wiederholte Prozeßführung, das Recht auf lange Sicht ökonomisch effizient machen soll ${ }^{26}$, wird in Wahrheit die Abweichung vom Pfad der Tugend lediglich in einer positiven Feedback-Schleife weiter verstärkt. Juridifizierung heißt Effizienzverlust!

Den Intentionen der regulatorischen Politik ergeht es nicht besser. „Mon code est perdu!“ Napoleon brach bekanntlich in Tränen aus, als er herausfinden mußte, daß sein berühmter code civil, der die neue freiheitliche Gesellschaft hervorbringen sollte, in die Hände sophistischer Anwälte und Richter und deren obskurer Hermeneutik geraten war. Aber dies ist generell das Schicksal regulatorischer Politik im Gerichtsprozeß. Die politische Festlegung regulatorischer Ziele, die planvolle Auswahl der jeweiligen Policyinstrumente und ihre auf Sozialbereiche abgestimmten Implementierungsstrategien werden im Zusammenspiel von Mandanten, Anwälten und Richtern für Prozessführungszwecke instrumentalisiert. So werden politische Programme ersetzt durch Strategien, mit denen man einen Rechtsstreit gewinnt. Zwangsläufig ändert sich, wenn policy-Instrumente zu Konfliktlösungsregeln transformiert werden, der Gehalt von Rechtsnormen.

Ein solcher Verlust der ursprünglichen „institutionellen“ Orientierung von Rechtsregeln ist, wenn sie in Gerichtsprozesse geraten, allen Typen von Produktionsregimes gemeinsam. Nicht nur die Vertragspraxis und die Gesetzgebung teilen dieses Schicksal, sondern auch Standardisierungs- und Normalisierungsprozesse, Marktregulierungen und Verwaltungsentscheidungen wie auch intra-organisatorische Regelsetzungen sind dieser inversen Entfremdung ausgesetzt. Es lassen sich vier Aspekte dieser Transformationsdynamik kennzeichnen. Erstens Zielverschiebung. Die Frage: Wer gewinnt, wer verliert den Prozess? ersetzt die Ursprungsorientierung der Norm, die etwa darin bestand, Effizienz zu steigern, technische Abläufe zu erleichtern, Verhalten zu regulieren oder soziale Institutionen zu stabilisieren. Zweitens Zeithorizont: Während die institutionelle Perspektive darauf gerichtet ist, zukunftsgerichtete Erwartungen im Produktionsregime zu stabilisieren, ist der Blick des Rechtsstreits in erster Linie auf die Vergangenheit gerichtet. In dem Maße, in dem vergangene Ereignisse im Gerichtssaal rekonstruiert werden, werden komplexe normative Strukturen auf die einzige Funktion reduziert: Welche Erwartung war im vorliegenden Fall die einzig „richtige“? Drittens Sprachwechsel: Während soziale Normen in der Sprache ihres institutionellen Kontexts formuliert sind, findet im Gerichtssaal unweigerlich ihre Übersetzung in die Rechtssprache statt. Die Frage lautet dann nur noch: Welche rechtlich geschützten Positionen gewähren sie den streitenden Parteien? Viertens Wirklichkeitskonstruktion: Während die Realitäten sozialer Normen vom cognitive mapping ihrer jeweiligen sozialen Institutionen bestimmt sind, zwingt der Rechtsstreit die Realitätskonstruktionen in die Zwei-Parteien-Perspektive des gerichtlichen Verfahrens. 
Dies Arrangement einer doppelten Rechtsentfremdung - Entfremdung vom Individualkonflikt und vom Produktionsregime - ist an sich wenig einleuchtend, dafür aber historisch um so erfolgreicher. Wie ist es zu erklären, daß die erzwungene Einheit inkongruenter Perspektiven zu einer solch stabilen institutionellen Konfiguration werden konnte. Sollte man nicht eher annehmen, daß die Dynamiken sozialer Ausdifferenzierung dieser Mesalliance längst ein Ende setzen? Auch wenn durchaus Tendenzen zu beobachten sind, daß sich innerhalb des Rechtssystems jeweils spezialisierte Schwerpunktorientierungen auf Konfliktlösung einerseits und Normproduktion andererseits herausbilden, bleibt die merkwürdige Tatsache, daß sich Gerichtsprozesse und Produktionsregimes weiterhin wechselseitig anziehen. Ja, es ist zu beobachten, daß sich die Anziehungskräfte in jüngster Zeit noch verstärken. Gerade im Bereich internationaler Beziehungen, wo die institutionalisierten Bahnen der Konfliktlösung und der Normproduktion seit Jahrhunderten getrennt verliefen, zeigt sich heute ein gradueller Verrechtlichungsprozeß, in dessen Zuge sich Produktionsregimes und (Quasi-)Gerichtsprozesse wechselseitig anziehen. ${ }^{27}$ Ähnliche Entwicklungen sind auf den internationalen Märkten auszumachen: in der Verrechtlichung des internationalen Vertragswesens und der internationalen Schiedsgerichtsbarkeit durch Institutionen der lex mercatoria und in der langsamen Transformation der WTO durch die Ausbildung gerichtsähnlicher Strukturen. ${ }^{28}$ In diesen Entwicklungen kann man beobachten, wie sich Konfliktmanagement und Produktionsregimes einander anziehen und zugleich einander sabotieren $^{29}$.

Zwei konkurrierende Interpretationen sind erkennbar. Die eine zielt auf Eigenwerte. Beide Prozesse, Konfliktresolution aufgrund normativer Erwartungen und Regelsetzung innerhalb der Produktionsregime, sind rekursive selbstreferentielle Prozesse, die je für sich allein gerade wegen ihrer Schließung in zirkulären Prozessen keine ausreichend stabilen Eigenewerte finden können, so daß sie unablässig auf der Suche nach äußeren Stabilisatoren sind. Zwar können soziale, wirtschaftliche und politische Prozesse über ihre internen Transaktionen protonormative Strukturen in den Produktionsregimes hervorbringen, sie sind aber nicht in der Lage, diese dauerhaft zu stabilisieren. Vielmehr werden diese volatilen Strukturen durch die gleichen Prozesse, also durch Änderungen der Machtlage, durch neue Marktstrukturen und durch Aufbau neuer sozialer Bindungen, die sie hervorgebracht haben, wieder unterminiert. In dieser Lage liegt es nahe, die protonormativen Strukturen ins Recht zu exportieren und sie dort durch das Symbol der Rechtsgeltung stabilisieren zu lassen. Auf diese Weise läßt sich in der Tat der unaufhörlichen Selbstzerstörung Einhalt gebieten. Damit nicht genug, denn das Ganze funktioniert auch in umgekehrter Richtung: Im Rahmen des gerichtlichen Verfahrens findet eine entsprechende Externalisierung der Normproduktion in der Weise statt, daß sich die interne Normherstellung auf externe „Autoritäten" - den Gesetzgeber, die Vertragsparteien, den Markt , formale Organisationen oder andere Institutionen - beruft. Damit verhindert sie unter anderem, daß ihre Geltung im Gerichtsproze B immer wieder aufs Neue in Frage gestellt wird. Ergebnis dieser Externalisierungen sind Eigenwerte, also relativ stabilen Normen für Produktionsregimes und akzeptierte Entscheidungskriterien für den Gerichtsprozeß, deren Inkongruität zugunsten von Erwartungsstabilisierung in Kauf genommen wird.

Die andere Interpretation zielt auf eine „Lock-in“-Situation. Sobald es zur gegenseitigen Anziehung von Prozeßführung auf der einen und Produktionsregimes auf der anderen Seite gekommen ist, entwickelt sich hier eine pfadabhängige evolutionärer Dynamik, die

28 Siehe jüngst etwa Lehmkuhl 2000; Frowein 2000; zum internationalen Vertragsrecht Döser 2000.

29 Dezalay/Garth 1995; Teubner 1996b. 
sie immer tiefer in ihre Obstruktionssymbiose treiben und sie darin festhält. Wenn ein Produktionsregime sich einmal der Dynamik gerichtlicher Prozeßführung geöffnet hat, gibt es keine Chance, auch nur irgendeinen seiner Bereiche vor dem Zugriff des Rechts zu schützen. Alle innerhalb eines Produktionsregimes entwickelten Normen unterliegen nun dem Risiko, „durchprozessiert“ und damit verfälscht zu werden. Und genauso ist es anders herum: Von dem Moment an, in dem sich Gerichte auf die Rechtssetzungsautorität externer Produktionsregimes berufen, können sie die Parteien und deren Anwälten nicht mehr daran hindern, andere dort produzierte Normen in den Prozeß einzuführen.

In der Tat fällt die Entscheidung zwischen den zwei Interpretationen - „Eigenwerte“ oder „Lock-in“ - schwer. Womöglich treffen sie beide zu, so daß sich hier Möglichkeiten empirischer Forschung abzeichnen, um den relativen Erklärungsgehalt beider zu erproben. Mehr noch ist zu erwarten von zukünftigen institutionellen Experimenten, die sich um Rationalitätssteigerung der Konfliktlösung und der Regelproduktion in den Produktionsregimes bemühen. Dann würde es leichter fallen, eine Grenze zwischen Eigenwerten und Lock-in zu ziehen. Dann könnte man auch besser einschätzen, ob Chancen bestehen, Konfliktlösung und Regelsetzung in Produktionsregimes jeweils kontextsensibler zu gestalten.

V.

Man kann sich des Eindrucks nicht erwehren, als sei hier eine „unsichtbare Hand“ am Werke, die strategisches Akteurshandeln in unbeabsichtigte soziale Wirkungen verwandelt. Aber von gemeinwohlsteigernden Wirkungen kann kaum die Rede sein. Eine kontextsensible und faire Lösung von Individualkonflikten ist nicht zu erwarten, allenfalls als Ergebnis der Anknüpfung an Produktionsregimes eine Gerechtigkeit inkongruenter Perspektiven. Ebensowenig sind kontextsensible und faire Rechtsnormen für das Produktionsregime zu erwarten, weil die unsichtbare Hand der Koevolution die soziale Normbildung durch den Filter von Gerichtsprozessen schickt, so daß letztlich die Dynamik eines Individualkonfliktes über die öffentlichen Regeln für Produktionsregimes entscheidet.

In der Sache geht es also um eine blinde Koevolution von rechtsstreitorientierter juristischer Semantik und institutionenorientierten sozialen Strukturen. Diese Koevolution garantiert nicht die soziale Adäquanz der Rechtsdogmatik gegenüber sozialen Institutionen, sondern verhindert sie. Hier stellt sich dann aber die Frage, wie es um die Chancen eines erneuten re-entry steht. Kann juristische Argumentation im Bewußtsein ihrer Doppelrolle in systematischer Weise soziales Wissen über Produktionsregimes mit dem Ziel entwickeln, die soziale Adäquanz der Rechtsnormen zu steigern?

Als geeignetes Beispiel mag hier die Problematik der sogenannten Drittwirkung von Grund- und Menschenrechten dienen. ${ }^{30}$ Das Eindringen der Medien in das Privatleben, die Verletzungen öffentlich-rechtlicher Verpflichtungen in privatisierten Unternehmen sowie diskriminierende Praktiken im Privatsektor machen die aktuelle Problematik mehr als deutlich. Was ist in diesem Bereich von einem re-entry sozialen Wissens in die Rechtsdogmatik zu erwarten?

Wegen ihres Engagement um latente Strukturen beansprucht die Gesellschaftstheorie mehr zu sehen als das Recht sieht, wenn Individuen Grundrechte geltend machen. Aus 
der Sicht der Gesellschaftstheorie geht es bei Grundrechten nicht bloß um den Schutz individueller Rechtspositionen, sondern um die Absicherung gesellschaftlicher Differenzierung. ${ }^{31}$ In knapper Skizzierung: Historisch gesehen entfalten sich individuelle Grundrechte im engen Zusammenhang mit der für moderne Gesellschaften typischen historischen Emergenz autonomer Handlungssphären. Sobald aber expansionistischen Tendenzen des politischen Systems beginnen, den Prozeß sozialer Ausdifferenzierung selbst zu unterminieren, kommt es zu politischen Auseinandersetzungen, in deren Zuge Grundrechte als soziale Gegeninstitutionen gerade dort entstehen, wo die soziale Differenzierung gegen die ihr inhärente Tendenz zur Selbstzerstörung geschützt werden soll. Individuelle Konflikte zwischen Privatbürger und Verwaltungsbürokratie dienen zugleich der rechtlich institutionalisierte Absicherung einer Selbstbeschränkung der Politik.

Was aber passiert, wenn die Latenz zerstört wird und Parteien, Anwälte und Richter sich ihrer bewußt werden, wenn also juristisches Argumentieren sich explizit auf eine andere Realität von Grundrechten einläßt? In diesem Moment wird die Diskrepanz zwischen individuellen Strategien und nicht-intendierten sozialen Ergebnissen offenkundig. Warum aber sollten individuelle Akteure zum Wohle sozialer Differenzierung einen Rechtsstreit führen? Eine Möglichkeit läge darin, die Verbindung zwischen den Individualmotiven zur Prozeßführung und den sozialen Wirkungen von Grundrechten zu stärken. Eine Chance hätte dies nur, wenn man eine stark partikularistische Komponente in die streng formelle und allgemeine Ausrichtung der Normen zur Sozialdifferenzierung einfügte. Die Aufgabe des Richters bestünde dann darin, in die rigiden Normen der Produktionsregimes eine starke Dosis von Partikularismus einzubringen. Um die genannte Diskrepanz zu überwinden, böte sich also an, individuelle Rechte im öffentlichen Interesse zu schaffen und in der Folge class actions, Verbandsklagen und andere Form privater Prozeßführung im öffentlichen Interesse zu ermöglichen. ${ }^{32}$

Andererseits wäre zu erwägen, Gesellschaftstheorie im Verfassungsrecht mit dem Ziel zu „verfeinern“, die hier geltenden Normen gegenüber den involvierten Produktionsregimes angemessener zu gestalten. ${ }^{33}$ Die Empfehlung lautet: Radikale Umstellung des verfassungsrechtlichen Arguments auf Institutionen- und/oder Systemtheorie! Aus der Sicht der ökonomischen Institutionentheorie ist ein Rechtssatz einem Produktionsregime dann adäquat, wenn es Individualkonflikte in der institutionellen Perspektive von Transaktionskostenminimierungen reinterpretiert. Dies wäre der ökomomisch motivierte Beitrag des Rechts zur Konstitutionalisierung von Produktionsregimes. Er liefe auf eine Stärkung von effizienten Organisations- und Marktstrukturen in den unterschiedlichsten Lebensbereichen hinaus. ${ }^{34}$ Aus der Sicht der Systemtheorie läge es dagegen nahe, Konflikten zwischen Akteuren in der Privatsphäre als Diskurskollisionen zu rekonstruieren. ${ }^{35}$ Das Ergebnis einer horizontalen Wirkung von Grundrechten bestünde dann nicht darin, sie unvermittelt auf Phänomene wirtschaftlicher Macht anzuwenden. Vielmehr ginge es darum, Grundrechte gegenüber solchen Kommunikationsmedien in Anschlag zu bringen, welche Kolonisierungstendenzen gegenüber anderen Bereichen des Soziallebens entfalten. Die Schutzfunktion der Grundrechte beschränkte sich daher nicht länger auf die Beziehung zwischen Bürger und Staat, sondern ihre Funktion als „Diskursrechte“ bezöge sich auf die expansiven Tendenzen von Sozialsystemen. Ihr normatives Korrelat bestünde darin,

\footnotetext{
$31 \quad$ Luhmann 1965; Willke 1975; Grimm 1987.

32 Zu class actions siehe jüngst Heß 2000.

33 Eine aufschlußreiche Diskussion von Sozialmodellen im Bürgerlichen Recht findet sich nun bei Wielsch 2000, Kapitel 3.

$34 \quad$ Vgl. besonders Williamson 1996a; 1996b.

35 Teubner 1996a; $1998 b$.
} 
Grundrechte in die Kontexte der private governance regimes zu erstrecken, zu welchen die Unternehmen, Medienkonzerne, Erziehungsinstitutionen, Berufs- und andere professionelle Verbände, sogenannte Quangos und internationale Organisationen zu zählen wären. Offensichtlich ist, daß dies eine grundsätzliche Transformation des klassischen Modells der Grundrechte in all seinen vier Elementen - Individuum, Staat, Macht, subjektives Recht - erfordert.

Dies also wären zwei Fälle des Imports von Gesellschaftstheorie in die Grundrechtsdogmatik, zum einen die Idee der Transaktionskostenminimierung und zum anderen die der Diskurskollisionen, die nun in der forensischen Praxis Anwendung finden sollen. Offensichtlich verhalten sich auch diese Kriterien wiederum orthogonal zum Einzelkonflikt. Aber es fragt sich, ob nicht die oben beschriebene Inkongruenz der Perspektiven kompensiert wird durch eine neue Sozialadäquanz des Rechts? Lassen sich nicht so Rechtsnormen entwickeln, die den Anforderungen effizienter Marktstrukturen ebenso angemessen sind wie denen einer polykontexturalen Gesellschaft?

Doch ist Skepsis gegenüber einer solchen Idee von Aufklärung des Rechts durch Gesellschaftstheorie angebracht. Denn die Probleme des re-entry sind auch hier nicht zu unterschätzen. „Sozialwissenschaften im Recht“ sind nichts anderes als eine weitere Form des re-entry der Recht/Nichtrecht-Unterscheidung in das Recht selbst, nur diesmal auf einer anderen Abstraktionsebene im Vergleich zu anderen re-entries: Normen/Fakten, Rechtsakten/gesellschaftlichen Handlungen, Rechtsbegriffen/Interessen. Auch hier bleibt man der unausweichlichen Verwirrung von internen Konstruktionen mit externer Realität ausgesetzt. Alle Versuche, Theorien durch direkten Rekurs auf die soziale Wirklichkeit zu testen, sind zum Scheitern verurteilt. Auch der radikale Konstruktivismus kann hier keinen gangbaren Ausweg aufzeigen. Wollte das Recht den radikalen Konstruktivismus tatsächlich ernst nehmen und inn in seine laufenden Operationen inkorporieren, würde dies unweigerlich zu seiner Lähmung führen. Der einzig gangbare Ausweg scheint in einer versuchsweisen und partiellen Selbst-Transparenz des rechtlichen re-entrys zu liegen. Am Phänomen formaler Organisation hat Luhmann den „Rationalitätsbegriff des durchschauten ,re-entry“" eingeführt:

"Die in sich wiedereintretende Unterscheidung (hier: von System und Umwelt) ist dieselbe und ist nicht dieselbe. Die 'Realität' ist immer nur als bezeichnete Realität, also nur dank referentieller Beobachtungen gegeben, aber sie ist nicht nur Referenz. Das gibt zumindest den Hinweis, daß das System sein Projektionsbewußtsein vorsichtig verallgemeinern, seine Selbstpsychiatrisierung normalisieren und so in einem strengen Sinne selbstkritisch werden kann, ohne sich den Boden der Realität unter den Füßen wegzuziehen. Es kann inn zu einem vorsichtig tastenden Gehen benutzen ohne die Illusion, man könne den richtigen Standort, das natürliche telos der Bewegung erreichen." 36

Das Recht sollte, gerade weil es sich über seine eigene Angemessenheit im Verhältnis zur Realitätskonstruktion nie sicher sein kann, mit unterschiedlichen Rechtsmodellen der sozialen Wirklichkeit experimentieren. Es hätte auf die Ergebnisse von Rechtsentscheidungen zu warten, die aus dem Experimentieren mit unterschiedlichen Realitätskonstruktionen hervorgehen, und darauf erneut zu reagieren. Mit anderen Worten: Der Widerstand 
einer Rechtskommunikation im Verhältnis zu einer anderen ist der einzig mögliche „Wirklichkeitstest“.

VI.

„Do Camels Have Standing?“ Wie das Recht mit anderen Annahmen über soziale Wirklichkeit experimentieren kann, dafür mögen die von Christopher Stone vehement geforderten Eigenrechte der Natur, die in jüngerer Zeit von Bruno Latour und Michel Ferres auch soziologisch und philosophisch abgestützt wurden ${ }^{37}$, als Beispiel dienen. Bedeutet dies den Eintritt in die new brave world der Ökologie? Geht es um Mother Gaia Autopoiesis als neue Kollektivakteurln? ${ }^{38}$ Oder befinden wir uns auf einer Reise zurück ins Mittelalter: „What Was It Like Try a Rat“? ${ }^{39}$ Können wir Naturobjekten Klagerechte zuerkennen? Oder handelt es sich nicht vielmehr um eine Ausbeutung der Natur durch das Recht zur Steigerung der Normproduktion? Diese Fragen betreffen in der Tat die Quantität und Qualität von Rechtssubjektivität und die Beziehung des Rechts zur natürlichen Umwelt. Wie dem auch sei, die ökologische Debatte hat jedenfalls die Frage neu aufgeworfen, welchen „lebenden Einheiten“ zu Recht der Status eines Rechtsakteurs zugesprochen werden kann.

In der Theoriesprache der Autopoiese wird das Problem reformuliert. Es geht nicht mehr darum, welche ontologischen Qualitäten (Intelligenz, Seele, reflexive Kapazitäten) eine Einheit besitzen muß, die sie dazu befähigen würden, ein Handlungssubjekt zu sein, sozial, rechtlich oder in anderer Weise. ${ }^{40}$ Stattdessen ist zu fragen: Unter welchen Bedingungen konstruiert ein Sozialsystem, also ein geschlossenes und autonomes Ensemble rekursiver Kommunikationen, das sich in der Umwelt der betroffenen Einheit befindet, das semantische Artefakt eines 'Akteurs'? Nicht die Einheit selbst, sondern das sie umgebende Sozialsystem bringt die Identität hervor, bescheinigt ihre Fähigkeit zur Handlung und Kommunikation, zur Wahrnehmung von Verantwortung, Rechten und Pflichten. Mit anderen Worten: Subjektivität ist keine natürliche Eigenschaft, sondern ein soziales Konstrukt. Das jeweilige Sozialsystem stattet seine Fiktionen mit Subjektivität aus. ${ }^{41}$ Als Mittel hierfür dient soziale Attribution. Eine ethnische oder territoriale Einheit etwa wird nicht deshalb zum Kollektivakteur ,Staat', weil sie bestimmte natürliche Eigenschaften oder eine spezifische organisatorische Form besitzt. Vielmehr ist ,Staat' das kommunikative Konstrukt des Systems internationaler Beziehungen, das seine Akteure hervorbringt und deren Teilnahme nur unter der Bedingung ermöglicht, daß ethnische und territoriale Einheiten die Form eines institutionalisierten Staates annehmen. ${ }^{42}$ Das Gleiche läßt sich von Wirtschaftsunternehmen sagen. Kollektivakteure sind sie nicht an sich, sondern als Ergebnis von Kommunikationsprozessen am Markt, ohne die sie nichts Weiteres wären als bloße Vertragsbündel. ${ }^{43}$

Nomen ossibus inhaeret. Wenn das Rechtssystem erst einmal dies alte Vorurteil über Bord geworfen hat und auch spirituelle Substanzen mit Namen ausgestattet und innen Rechte verliehen hat, kann es selbst an gänzlich andere Konfliktdynamiken anschließen, die es inm erlauben, seine Normproduktion deutlich zu steigern. Die Erfindung der juristischen Person muß in der Tat als der große kulturelle Beitrag des Rechts zur organisatori-

Stone 1972; Latour 1998; 1994, Kap. 5; Serres 1990.

Lovelock 1979.

Ewald 1995; Amira 1891.

Luhmann 2000, Kapitel 13.

Luhmann 1984, 270 ff.; Teubner 1987.

Luhmann 1998.

Teubner 1992. 
schen Revolution der Neuzeit angesehen werden, bei der die Zuschreibung von Handlungsfähigkeit von Menschen auf Kommunikationsprozesse ausgeweitet wurde. Mit Hilfe der zunächst äußerst unwahrscheinlichen Idee, Rechtspersönlichkeit nicht nur an Individuen, sondern auch an Kommunikationsflüsse zu verleihen, erschuf das Recht ein weiteres Kamel, lieh es der Gesellschaft aus und profitierte selbst davon in größter Weise, als die Gesellschaft auch ihrer Rückgabepflicht genügte.

Hier geht es in erster Linie um formale Organisationen als Kollektivakteure. Sobald Organisationen die Rechtspersönlichkeit verliehen wurde, indem innen Klagerechte zugedacht wurden, konnte das Recht das Konfliktpotential organisierter Handlungen für seine eigene Normproduktion in enormer Weise ausbeuten. Im Vergleich hierzu mußte das Potential individueller Konflikte zweifellos in den Schatten treten ${ }^{44}$. „Why the Haves Come Out Ahead" - unter diesem Slogan haben Rechtssoziologen den großen Unterschied hinsichtlich des Konfliktpotentials von Organisationen und Individuen im Gerichtssaal untersucht. ${ }^{45}$ Dies betrifft jedoch nur einen Teilaspekt. Selbstverständlich haben Kollektivakteure regelmäßig andere Chancen als Individuen, aus einem Rechtsstreit als Gewinner hervorzugehen. Eine genaue empirische Untersuchung der Rolle von Organisationen in Gerichtsprozessen und ihre Bedeutung für die rechtliche Normenproduktion steht jedoch noch aus, besonders in Hinsicht auf die Koevolution von juristischer Semantik auf der einen und formaler Organisation auf der anderen Seite.

Die ökologische Bewegung hat nun das Vorurteil, wonach nur menschliche Individuen Akteure sein können, erneut erschüttert. Wie aber soll man die „Aktanten“ in der Neuen Politischen Ökologie identifizieren und gar rechtlich qualifizieren? Umweltschutzgruppen selbst scheinen bislang die einfachsten Fälle „neuer" Akteure zu sein. Aber was ist mit zukünftigen Generationen, bestimmten Tierarten, Pflanzen, Landschaften? Wie steht es mit Sprachen? Kulturen? Es stellt sich die Frage, ob es sich hier lediglich um neue Akteure im Rahmen des ökologischen Diskurses handelt, die für ihre Interessen und Rechte kämpfen und damit für ihre formelle Anerkennung als Rechtsakteure, oder ob hier nicht vielmehr die Ankopplung des Rechtsprozesses an andere "lebende“ Prozesse betroffen ist. Dies hätte freilich zur Folge, dass seine Normenproduktion in ganz neue Richtungen gelenkt würde.

Niklas Luhmann, ebenso wie Bruno Latour und Michel Serres behaupten in der Tat, daß sie solche ökologischen Kamele schon beobachtet haben. Heißt dies, daß Subjektivität, ungeachtet dessen, ob dies nun Menschen, formale Organisationen, politische Staaten oder andere Akteure betrifft, einfach auf ihren Status rechtlicher Fiktionen reduziert ist, auf ihre Eigenschaft als kommunikative Artefakte? Davon kann schon deswegen keine Rede sein, weil Sozialsysteme hinsichtlich ihrer Akteursattribution höchst selektiv verfahren. Die Zurechnung erfolgt nur unter der Bedingung: Die Seele kehrt in die Gesellschaft zurück. Nicht wirklich, sondern wieder im ambivalenten Sinne eines re-entry. Bevor Sozialsysteme kommunikativen Objekten Subjektivität verleihen, seien dies nun Menschen, Kollektive, geistige Substanzen, Engel, Tiere oder Computer, verlangen sie glaubhafte Indikatoren einer Adressabilität. ${ }^{46}$ Subjektivität wird nur unter der Bedingung verliehen, daß es für das Sozialsystem genügend gute Gründe zu der Realitätsunterstellung (!) gibt, daß sich „hinter" den sozialen Adressen selbstreferentielle Verstehensprozesse und Kommunikationspotentiale verbergen. Gleichzeitig verlangen sie eine enge strukturelle Kopplung mit ihren

\footnotetext{
$44 \quad$ Hutter/Teubner 1994.

45 Galanter 1974; Röhl 1987.

$46 \quad$ Fuchs 1991; 1997.
} 
eigenen Kommunikationsprozessen. Soziale Systeme verleihen Subjektivität also nur wenn sie (1) Grund haben, Verstehensoperationen hinter ihren kommunikativen Artefakten zu unterstellen, wenn sie (2) annehmen können, daß diese Artefakte dasselbe bei ihren Partnern unterstellen und (3), wenn das Subjektivität verleihende Sozialsystem selbst eine interne Irritabilität im Verhältnis zu den Beiträgen dieser "Subjekte“ entwickelt hat. In ihrer operationellen Geschlossenheit haben Sozialsysteme keinen Zugang zur selbstreferentiellen Realität des Anderen, gleichzeitig müssen sie jedoch in ihren internen Strukturen darauf vorbereitet sein. Nicht anders als der ungläubige Thomas müssen sie irritiert werden können, bevor sie an die Existenz des Anderen zu glauben beginnen.

Für formale Organisationen heißt das etwa, daß sie nur dann zu Kollektivakteuren werden können, wenn das sie umgebende Sozialsystem gute Gründe hat, an ihre unsichtbare Selbstreferentialität zu glauben. Das Sozialsystem muß also einen bestehenden geschlossenen selbstreferentiellen Signifikationsprozess unterstellen können, daneben das Vorhandensein von ausgebildete Entscheidungsrollen und Verfahren zur kollektiven Repräsentation und es muß sich schließlich durch die Dynamiken organisatorischer Prozesse tatsächlich irritiert „fühlen“. So werden ethnische Einheiten vom internationalen Recht auch erst dann als Staaten anerkannt, wenn dieses in „,̈berwältigender“ Weise von der Existenz der drei berühmten Bedingungen von Staatlichkeit überzeugt ist: Territorium, Volk, Herrschaftssystem. Wie aber steht es mit Latours ökologischen Aktanten? Latour stellt zutreffend fest, daß der historische Beitrag der ökologischen Bewegung in der Gründung der "septième cité" führt. Ausgehend von Boltanskis und Thévenots Theorem der sechs geschlossenen und inkompatiblen Rechtfertigungsdiskurse ${ }^{47}$ stellt Latour die These auf, daß der neue Diskurs der Politischen Ökologie das Potential besitzt, ein vollständiges neues Sozialsystem zu gründen, eine „grüne Stadt“, die von der Spezies ökologischer Aktanten bevölkert ist.
„Political ecology ... bears on complicated forms of associations between be- ings: regulations, equipment, consumers, institutions, habits, calves, cows, pigs. ... a collective experimentation on the possible associations between things and people .... a network of quasi-objects whose relations of subordi- nation remain uncertain and which thus require a new form of political activ- ity adapted to following them ${ }^{48}$

Wie dem auch sei, im „Parlament der Dinge“ haben nur einige von Latours Aktanten, jedenfalls nicht alle, Chancen auf soziale und rechtliche Anerkennung. Mit guten Gründen betont Latour daher, daß es auf die Anerkennung der Autonomie von Prozessen ankomme, weil nur auf dieser Grundlage über den Akteurstatus entschieden werde. Dennoch können wir Latour symmetrischer Anthropologie nicht folgen. ${ }^{49}$ Wir sind, wir waren und wir bleiben modern und können gar nicht anders. So ist in heutigen Sozialsystemen nicht zu erwarten, daß Engel, Tempel, Götter und Orakel, die in bestimmten Gesellschaften reale Subjekte sozialer Kommunikation waren, irgendwann in der Zukunft wieder soziale Akteure sein werden, nicht aber, weil sie nicht existieren - wer weiß? -, sondern weil moderne Sozialsysteme ihre Irritabilität innen gegenüber verloren haben. In den Realitätskonstrukten dieser Sozialsysteme ist die soziale Welt nicht länger durchsetzt von

\footnotetext{
$47 \quad$ Boltanski/Thévenot 1991.

48 Latour 1998, 229, $234 \mathrm{f}$.

$49 \quad$ Latour 1994.
} 
„nicht-menschlichen Adressen, mit Kommunikationschancen, die sich auf Ahnengeister, Götter, Bäume, heilige Schreine, Eingeweide, Vogelflug bezogen, auf alle sichtbaren und unsichtbaren Phänomene, denen ein Umgang mit eigener Selbstreferenz unterstellt werden konnten (also auch die Möglichkeit, zu lügen, zu täuschen, zu ,trickstern“ und ,willentlich‘ zu schweigen. “50

Und dennoch: Ökologische Einheiten, soziotechnische Artefakte, Cyborgs, Hybride, Netzwerke und andere informationsverarbeitende latourianische Aktanten haben eine reale Chance, die moderne Gesellschaft zu irritieren und an den Konfliktprozessen der Neuen Politischen Ökologie teilzunehmen.

Und das Kamel des Richters? Die Zeichen mehren sich, daß das Recht beginnt, seine prozeduralen und konzeptuellen Apparaturen auf die Herstellung neuer fiktiver Bewohner der "septième cité" einzustellen. Die Aufnahme von Umweltrechten in Verfassungstexte, die graduelle Anerkennung von Rechten für Tiere, die Veränderungen in der juristischer Sprache von einer Naturschutzsemantik über den Schutz ökologischer Interessen bis hin zu Eigenrechten von Lebensprozessen, der langwierige Prozeß, Umweltgruppen Klagerechte oder wenigstens Prozeßbeteiligungschancen zu verleihen und die sich ausweitende Konzeptualisierung von impersonalen Umweltschäden sind allesamt Indikatoren dafür, daß das Recht auf dem besten Wege ist, der Gesellschaft eine neue Spezies von Kamelen zur Verfügung zu stellen. ${ }^{51}$ „Aktanten“ und „Mediatoren“ in diesem sich herausbildenden ökologischen Diskurs bedürfen keiner vollständigen Rechtssubjektivität, um eine neue politische Dynamik zu entfalten. Zahlreiche juristische Unterscheidungen - etwa zwischen unterschiedlichen Abstufungen von Rechtssubjektivität, zwischen geschützten Interessen, partiellen und umfassenden subjektiven Rechten, zwischen beschränkter und unbeschränkter Handlungsfähigkeit, zwischen unterschiedlichen Formen der Interessenrepräsentation, zwischen individueller Haftung, gesamtschuldnerischer Haftung, Unternehmenshaftung, Konzernhaftung, market share liability - und anderen Formen kollektiver Verantwortung - bergen das Potential, Konfigurationen ökologischer Aktanten einen sorgfältig umrissenen Rechtsstatus zu verleihen. Diese Realfiktionen werden ihrer Rolle wahrscheinlich nur innerhalb des ökologischen Diskurses spielen, ohne notwendigerweise als Akteure überall sonst in der Gesellschaft aufzutreten. Rechtsfähigkeiten können durchaus selektiv in unterschiedlichen Sozialkontexten zugeschrieben werden. Dann stehen in der Tat die Chancen nicht schlecht, daß die Richterkamele auch in Zukunft fruchtbar sind und sich vermehren.

\section{Literatur}

Amira, Karl v. 1891: Thierstrafen und Thierprozesse. Innsbruck: Wagner.

Baecker, Dirk 1993: Die doppelte Schließung der Organisation. In: Teoria Sociologica 2, S. 147-184.

Becker, Dietrich 2000: Zur Geschichte der §§ 2088, 2089 BGB - Einige Anmerkungen. In: http://www.rz.uni-frankfurt.de/FB/fb01/teubner.

Boltanski, Luc /Thévenot, Laurent 1991: De la justification: Les économies de la grandeur. Paris: Gallimard.

Bourdieu, Pierre 1986: La force du droit: Elements pour une sociologie du champ juridique. In: Actes de la recherche en sciences sociales, S. 3-19.

Erbel 1986; Schmidt 1996; Pfordten 1995; Godt 1997; Ogorek 1999. 
Christie, Nils 1976: Konflikt als Eigentum. In: Informationsbrief der Sektion Rechtssoziologie derdeutschen Gesellschaft für Soziologie 12, S. 12.

Classen, Claus Dieter 1997: Drittwirkung der Grundrechte in der Rechtsprechung des Bundesverfassungsgerichts. In: Archiv des Öffentlichen Rechts 122, S. 65-107.

Cooter, Robert/Kornhauser, Lewis 1980: Can Litigation Improve the Law without the Help of Judges? In: Journal of Legal Studies 9, S. 139-163.

Cotterrell, Roger B. M. 1995: Law's Community: Legal Theory in Sociological Perspective. Oxford: Oxford University Press.

Dezalay, Yves/Garth, Bryant 1995: Merchants of Law as Moral Entrepreneurs. In: Law \& Society Review 29, S. 12-27.

Döser, Wulf H. 2000: Einführung in die Gestaltung internationaler Wirtschaftsverträge. In: Juristische Schulung, S. 246-254, 456-459.

Erbel, Günter 1986: Rechtsschutz für Tiere. In: Deutsches Verwaltungsblatt, S. 1235-

Esposito, Elena 1993: Ein zweiwertiger nicht-selbständiger Kalkül. In: Dirk Baecker

(Hrsg.), Kalkül der Form. Frankfurt: Suhrkamp, S. 96-111.

Ewald, William 1995: Comparative Jurisprudence (I): What Was It Like to Try a Rat. In: University of Pennsylvania Law Review 143, S. 1889-2149.

Fitzpatrick, Peter 1992: The Impossibility of Popular Justice. In: Social \& Legal Studies 1, S. $199-215$.

Foerster, Heinz v. 1993: Wissen und Gewissen. Frankfurt : Suhrkamp.

Frowein, Jochen A. 2000: Konstitutionalisierung des Völkerrechts. In: Völkerrrecht und Internationales Privatrecht in einem sich globalisierenden internationalen System Auswirkungen der Entstaatlichung transnationaler Beziehungen. Berichte der Deutschen Gesellschaft für Völkerrecht 39. Heidelberg: C.F. Müller 2000, 427-447.

Fuchs, Peter 1991: Kommunikation mit Computern? Zur Korrektur einer Fragestellung. In: Scoiologia Internationalis 29, S. 1-30.

Fuchs, Peter 1996: Die archaische Second-Order-Society: Paralipomena zur Konstruktion der Grenze der Gesellschaft. In: Soziale Systeme 2, S. 113-130.

Fuchs, Peter 1997: Adressabilität als Grundbegriff der soziologischen Systemtheorie. In: Soziale Systeme 3, S. 57-79.

Galanter, Marc 1974: Why the 'Haves' Come Out Ahead: Speculations on the Limits of Legal Change. In: Law \& Society Review 9, S. 95-160.

Godt, Christine 1997: Haftung für ökologische Schäden: Verantwortung für Beeinträchtigungen des Allgemeingutes Umwelt durch individualisierende Verletzungshandlungen. Berlin: (?).

Graber, Christoph/Teubner, Gunther 1998: Art and Money: Constitutional Rights in the Private Sphere. In: Oxford Journal of Legal Studies 18, S. 61-74.

Grimm, Dieter 1987: Grundrechte und Privatrecht in der bürgerlichen Sozialordnung. In: Dieter Grimm, Recht und Staat in der bürgerlichen Gesellschaft. Frankfurt : Suhrkamp, S. 192-211.

Günther, Klaus 1988: Der Sinn für Angemessenheit: Anwendungsdiskurse in Moral und Recht. Frankfurt : Suhrkamp.

Habermas, Jürgen 1992: Faktizität und Geltung. Frankfurt: Suhrkamp.

HeB, Burkhard 2000: Die Anerkennung eines Class Action Settlement in Deutschland. IN: Juristenzeitung 55, S. 373-382.

Higgins, Rosalyn 1997: The Reformation in International Law. In: Richard Rawlings (Hrsg.), Law, Society and Economy. Oxford: Oxford University Press, S. 207-224.

Hunt, Murray 1998: The 'Horizontal Effect' of the Human Rights Act. In: Public Law, S. 423-443.

Hutter, Michael/ Teubner, Gunther 1994: Der Gesellschaft fette Beute. Homo juridicus und homo oeconomicus als kommunikationserhaltende Fiktionen. In: Peter 
Fuchs/Andreas Göbel (Hrsg.), Der Mensch - das Medium der Gesellschaft?. Frankfurt: Suhrkamp, S. 110-145.

Kelman, Mark 1987: A Guide to Critical Legal Studies. Cambridge, Mass.: Harvard University Press.

Kennedy, Duncan 1997: Critique of Adjudication. Cambridge, Mass.: Harvard University Press.

Kerchove, Michel v. d./Ost, François 1992: Le droit ou les paradoxes du jeu. Paris : Presses Universitaires de France.

Latour, Bruno 1994: Wir sind nie modern gewesen: Versuch einer symmetrischen Anthropologie. Berlin : Akademie Verlag.

Latour, Bruno 1998: To Modernise or to Ecologise? That is the Question. In: Bruce Braun/Noel Castree (Hrsg.), Remaking Reality: Nature at the Millenium. London : Routledge \& Paul, S. 221-242.

Lehmkuhl, Dirk 2000: Commercial Arbitration - A Case of Private Transnational SelfGovernance?, Preprints aus der Max-Planck-Projektgruppe Recht der Gemeinschaftsgüter, Bonn (2000/1).

Lovelock, J. E. 1979: Gaia: A New Look at Life on Earth. Oxford: Oxford University Press.

Luhmann, Niklas 1965: Grundrechte als Institution: Ein Beitrag zur politischen Soziologie. Berlin : Duncker \& Humblot.

Luhmann, Niklas 1975: Interaktion, Organisation, Gesellschaft. In: Niklas Luhmann, Soziologische Aufklärung 2. 3. Aufl. 1986. Opladen : Westdeutscher Verlag, S. 9-20.

Luhmann, Niklas 1984: Soziale Systeme: Grundriß einer allgemeinen Theorie. Frankfurt : Suhrkamp.

Luhmann, Niklas 1993a: Observing Re-entries. In: Graduate Faculty Philosophy Journal 16, S. 485-498.

Luhmann, Niklas 1993b: Das Recht der Gesellschaft. Frankfurt/M.: Suhrkamp.

Luhmann, Niklas 1995: Juristische Argumentation: Eine Analyse ihrer Form. In: G. Teubner (Hrsg.), Entscheidungsfolgen als Rechtsgründe: Folgenorientiertes Argumentieren in rechtsvergleichender Sicht. Baden-Baden : Nomos, 19-37.

Luhmann, Niklas 1997: Die Gesellschaft der Gesellschaft. Frankfurt/M.: Suhrkamp.

Luhmann, Niklas 1998: Der Staat des politischen Systems: Geschichte und Stellung in der Weltgesellschaft. In: Ulrich Beck (Hrsg.), Perspektiven der Weltgesellschaft. Frankfurt: Suhrkamp, S.. 345-380.

Luhmann, Niklas 2000: Organisation und Entscheidung. Opladen: Westdeutscher Verlag (im Erscheinen).

Maturana/Varela 1987: Der Baum der Erkenntnis: Die biologischen Wurzeln menschlichen Erkennens. Bern: Scherz

Nelson, Richard R. Winter, Sidney G. 1982: An Evolutionary Theory of Economic Change. Cambridge, Mass.: Harvard University Press.

Oeter, Stefan 1994: „Drittwirkung“ der Grundrechte und die Autonomie des Privatrechts, Archiv des Öffentlichen Rechts 119, S. 529-563.

Ogorek, Regina 1999: Recht und Tier: Eine traurige Begegnung. In: Rechtshistorisches Journal 18, S. 247-259.

Pfordten, Dietmar v. d. 1995: Die moralische und rechtliche Berücksichtigung von Tieren. In: J. Nida-Rümelin/D. von der Pfordten (Hrsg.), Ökologische Ethik und Rechtstheorie. Baden-Baden : Nomos, S. 231-244.

Röhl, Klaus 1987: Die strukturelle Differenz zwischen Individuum und Organisation oder Brauchen wir ein Sonderprivatrecht für Versicherungen und andere Organisationen? In: Festschrift für Ernst C. Stiefel. München: Beck, S. 574-605. 
Schmidt, Thomas B. 1996: Das Tier - ein Rechtssubjekt? Eine rechtsphilosophische Kritik der Tierrechtsidee. Regensburg : Roderer.

Schmitt, Carl 1934: Politische Theologie (1922). München/Leipzig: Duncker \& Humblot, 2. Aufl.

Schmitt, Carl 1963: Der Begriff des Politischen (1932). Berlin: Duncker \& Humblot.

Serres, Michel 1990: Le contrat naturel. Paris: Flammarion.

Soskice, David 1997: Divergent Production Regimes: Coordinated and Uncoordinated Market Economies in the 1980s and 1990s. In: H. Kitschelt/P. Lange/G. M. a. J. D. Stephens (Hrsg.), Continuity and Change in Contemporary Capitalism. Cambridge : Cambridge University Press, S. 271-289.

Spencer Brown, George 1997: Gesetze der Form. Lübeck : Bohmeier.

Stone, Christopher D. 1972: Should Trees Have Standing? - Toward Legal Rights for

Natural Objects. In: Southern California Law Review 45, S. 450.

Teubner, Gunther 1987: Unternehmenskorporatismus: New Industrial Policy und das Wesen der Juristischen Person. In: Kritische Vierteljahresschrift für Gesetzgebung und Rechtswissenschaft 2, S. 61-85.

Teubner, Gunther 1989: Recht als autopoietisches System. Frankfurt : Suhrkamp.

Teubner, Gunther 1992: Die vielköpfige Hydra: Netzwerke als kollektive Akteure höherer Ordnung. In: W. Krohn/G. Küppers (Hrsg.), Emergenz: Die Entstehung von Ordnung, Organisation und Bedeutung. Frankfurt : Suhrkamp, S. 189-216.

Teubner, Gunther 1995: Die zwei Gesichter des Janus: Rechtspluralismus in der Spätmoderne. In: Eike Schmidt/Hans-Leo Weyers (Hrsg.), Liber Amicorum für Josef Esser. Heidelberg : Müller, S. 191-214.

Teubner, Gunther 1996a: Altera pars audiatur: Das Recht in der Kollision unterschiedlicher Universalitätsansprüche. In: Archiv für Rechts- und Sozialphilosophie, S. 199220.

Teubner, Gunther 1996b: Globale Bukowina: Zur Emergenz eines transnationalen Rechtspluralismus. In: Rechtshistorisches Journal 15, S. 253-255.

Teubner, Gunther 1998a: Legal Irritants: Good Faith in British Law or How Unfying Law Produces New Divergences. In: Modern Law Review 61, S. 11-32.

Teubner, Gunther 1998b: Verragswelten: Das Recht in der Fragmentierung von Private Governance Regimes. In: Rechtshistorisches Journal 17, S. 234-265.

Teubner, Gunther 1999a: Eigensinnige Produktionsregimes. In: Soziale Systeme 5, S. 725.

Teubner, Gunther 1999b: Ökonomie der Gabe - Positivität der Gerechtigkeit: Gegenseitige Heimsuchungen von System und différance. In: Albrecht Koschorke/Cornelia Vismann (Hrsg.), System - Macht - Kultur: Probleme der Systemtheorie. Berlin: Akademie.

Varela, Francisco J. 1992: Whence Perceptual Meaning? A Cartography of Current Ideas. In: F. J. Varela/J. P. Dupuy (Hrsg.), Understanding Origins: Contemporary Views on the Origin of Life, Mind and Society. Dordrecht: Kluwer, S. 235-263.

Vesting, Thomas 1994: Von der liberalen Grundrechtstheorie zum Grundrechtspluralismus. In: Christoph Grabenwarter/Stefan Hammer/Alexander Pelzl/Eva SchulevSteindl/Ewald Wiederin (Hrsg.), Allgemeinheit der Grundrechte und Vielfalt der Gesellschaft. Stuttgart u.a., S. 9-24.

Weick, Karl E. 1979: The Social Psychology of Organizing. Reading : Addison-Wesley. Wielsch, Dan 2000: Freiheit und Funktion: Zur Struktur- und Theoriegeschichte des Rechts der Wirtschaftsgesellschaft. Baden-Baden: Nomos (im Erscheinen) Williamson, Oliver 1996a: The Mechanisms of Governance. Oxford : Oxford University Press. 
Williamson, Oliver E. 1996b: Vergleichende ökonomische Organisationstheorie: Die Analyse diskreter Strukturalternativen. In: Patrick Kenis/Volker Schneider (Hrsg.), Organisation und Netzwerk: Institutionelle Steuerung in Wirtschaft und Politik. Frankfurt: Campus, S. 213-273.

Willke, Helmut 1975: Stand und Kritik der neueren Grundrechtstheorie: Schritte zu einer normativen Systemtheorie. Berlin: Duncker \& Humblot. 